\title{
Monitoring Journal Bearing Using Acoustic Emission (AE) Technique
}

\author{
R.I. Raja Hamzah, S.Husin
}

This paper presents the measurement procedures and the results of the Acoustic Emission (AE) assessment on ID Fan journal bearings. Vibration data were also acquired in an effort to compare the sensitivity of both techniques in detecting incipient failures. Series of Acoustic Emission (AE) and vibration measurements have been undertaken on the selected journal bearings for ID fan at power plant. AE and vibration statistical parameters were calculated from the collected raw data in an effort to compare the sensitivity of both techniques in detecting incipient failures. The purpose of the measurements is to evaluate the capability of $\mathrm{AE}$ system in detecting incipient failures in journal bearing. This report details the measurements procedures undertaken and the results of this assessment. 